\title{
Locally advanced squamous cell cervical cancer in a patient with septate uterus
}

\author{
Guler Yavas, MD, Cagdas Yavas, MD, Gokcen Inan, MSc \\ Department of Radiation Oncology, Selcuk University, Konya, Turkey
}

\begin{abstract}
A 33-year-old premenopausal multiparous woman with the history of septate uterus was referred to the hospital with menorrhagia and inter-menstrual bleeding occurring for the last 6 months. Work-up revealed a stage IIB cervical cancer. The patient underwent curative chemo/radiotherapy. The patient's septate uterus presented several difficulties during application. Brachytherapy was applied using standard computed tomography-compatible tandem and ovoids. The high-risk clinical target volume (HR-CTV), intermediate risk CTV, and organs at risk were contoured according to the Groupe Européen de Curiethérapie - European Society for Radiology and Oncology (GEC-ESTRO) guidelines. Treatment was performed via 3-dimensional high-dose-rate technique with ${ }^{192} \mathrm{Ir}$, with brachytherapy dose of 28 Gy in 4 fractions/7 Gy each, prescribed to the HR-CTV. Treatment was well tolerated with manageable acute toxicities. To the best of our knowledge, the current case is the second case of septate uterus with locally advanced cervical carcinoma. Due to the lack of guidelines about brachytherapy applications in patients with uterine anomaly and owing to the anatomical anomaly, brachytherapy application is challenging in this patients population.
\end{abstract}

J Contemp Brachytherapy 2017; 9, 5: 487-489

DOI: https://doi.org/10.5114/jcb.2017.70996

Key words: brachytherapy, cervical cancer, septate uterus, uterine anomalies.

\section{Purpose}

The standard management of individuals with locally advanced cervical cancer includes external pelvic radiotherapy (EPRT) with concurrent cisplatin-based chemotherapy with brachytherapy (BRT) [1,2]. Brachytherapy is critical for curative-intent treatment of cervical cancer, because it has intrinsic physical property of steep dose gradient, and has a potential to deliver relatively large dose while sparing the surrounding organs at risk (OAR) [3,4], and when it is replaced with EPRT, the results are clearly inferior $[5,6,7]$. The skills and expertise of the radiation oncologist and proper applicator placement are critical determinants of dose specification in addition to the treatment success in BRT $[8,9,10,11]$. The American Brachytherapy Society (ABS) and the Groupe Européen de Curiethérapie - European Society for Radiology and Oncology (GEC-ESTRO) working group, have released specific guidelines to aid radiation oncologist in performing effective and safe BRT application in cervical cancer $[8,12,13]$.

Uterine anomalies occur in 2 to 4 percent of fertile women with normal reproductive outcomes [14,15]. Among all the uterine anomalies, the septate uterus frequency was reported as a $35 \%$ in a review [15]. Herein, we report an unusual case of a patient with a congenital septate uterus who received definitive EPRT and concom- itant cisplatin, followed by 3-dimesional BRT for locally advanced cervical cancer.

\section{Case presentation}

A 33-year-old premenopausal multiparous woman was referred to the hospital with menorrhagia and intermenstrual bleeding occurring for the last 6 months, with a history of septate uterus (Figure 1). Gynecological examination revealed a bulky cervical tumor with right parametrial invasion and extension to the upper part of the vagina. The biopsy of this lesion revealed a squamous cell cervical cancer. Cystoscopy and rectoscopy were normal. A pelvic magnetic resonance imaging (MRI) found a $42 \times 55 \times 60 \mathrm{~mm}$ enhancing cervical mass arising from the cervix with a parametrial extension on the right side. Whole body positron emission tomography/computed tomography (PET-CT) scan revealed high fluorodeoxyglucose uptake in primary cervical tumor and right internal iliac lymph node with a maximum standardized uptake value $\left(\mathrm{SUV}_{\text {max }}\right)$ of 25.73. According to the Federation Internationale de Gynecologic et d'Obstetrique (FIGO) classification, the tumor was stage IIB.

The patient underwent laparoscopic extraperitoneal lymph node dissection and curative chemoradiotherapy. External pelvic radiotherapy was performed using 3-dimensional conformal radiotherapy, with four-field tech- 


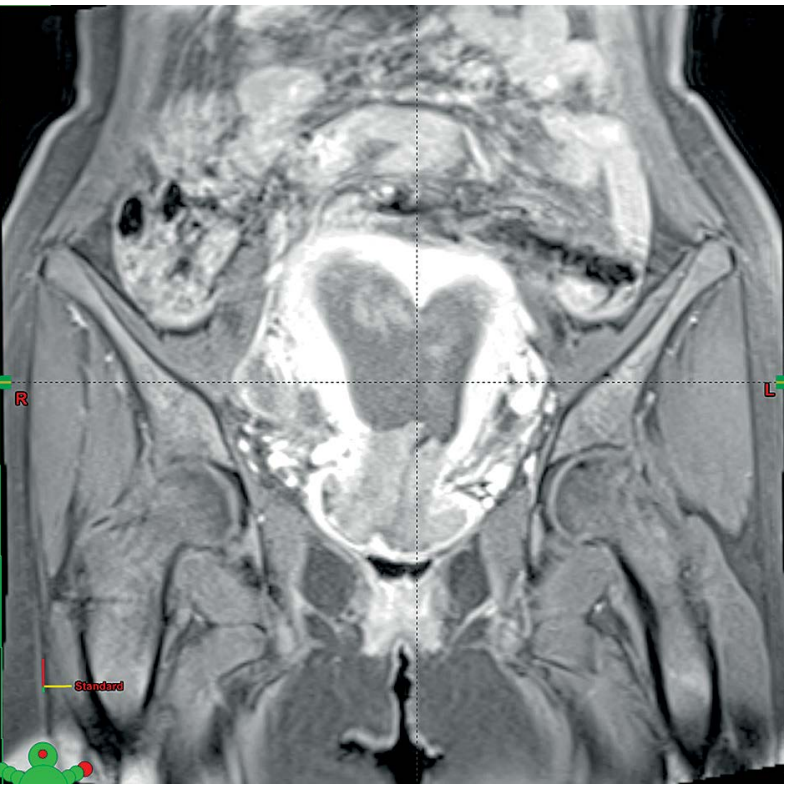

Fig. 1. Magnetic resonance images in the coronal plane demonstrating a septate uterus

nique to a dose of $45 \mathrm{~Gy}$ in 25 daily fractions of $1.8 \mathrm{~Gy}$ each with the use of midline blocks up to $50.4 \mathrm{~Gy}$. Throughout the treatment, the patient received weekly cisplatin at a dose of $40 \mathrm{mg} / \mathrm{m}^{2}$. Treatment could be given without interruption. The patient was evaluated on fraction 20 of EPRT; however, we could not perform BRT application because of the remaining exophytic tumor. Brachytherapy application could be performed after EPRT. Since the patient denied general anesthesia, the BRT application was performed under sedation with intramuscular midazolam. Without using general anesthesia, it was very difficult to perform the application. Therefore, we used vaginal misoprostol. Since the patient's septate was located superiorly and the left side of the uterus was very small when compared to right side, we could employ standard uterine tandem to the right side. The tumor was also located on the right side predominantly. After successfully placing the tandem, two CT-compatible ovoids were placed in right and left vaginal fornices. Once the tandem and the ovoids were successfully placed and appropriate geometry obtained, vaginal packing was completed to separate rectum and bladder. Computed tomography was performed for treatment planning. High-risk clinical target volume (HR-CTV), intermediate risk CTV (IR-CTV), and OAR were contoured. Iridium-192 ( $\left.{ }^{192} \mathrm{Ir}\right)$ high-dose-rate (HDR) brachytherapy treatment (GammaMedplus ${ }^{\mathrm{TM}}$ iX HDR Brachytherapy Afterloader, Varian, USA) was delivered, with 28 Gy in 4 fractions over 2 weeks.

Organs at risk constraints were calculated using total equieffective dose $\left(\mathrm{EQD}_{2}\right)$ according to GEC-ESTRO recommendations $[13,16]$. Coverage for the HR-CTV was analyzed using recommended dose-volume histogram (DVH) parameter of $D_{90}$, which was greater than 7 Gy for each fraction of BRT. Dose limits to the normal structures were within acceptable limits for entire treatment: bladder $\mathrm{D}_{2 \mathrm{cc}}=76.85 \mathrm{~Gy}_{\mathrm{EQD} 2}$, rectum $\mathrm{D}_{2 \mathrm{cc}}=71.93 \mathrm{~Gy}_{\mathrm{EQD} 2}$ and sigmoid $\mathrm{D}_{2 \mathrm{cc}}=69.75 \mathrm{~Gy}_{\mathrm{EOD} 2}$. Total $\mathrm{D}_{90}$ to the HR-CTV was calculated as $84.35 \mathrm{~Gy}_{\mathrm{EQD} 2}$. Total treatment time (EPRT plus BT) was 53 days.

\section{Discussion}

The development of female urogenital tract in utero starts around the sixth week of embryological life. Three main structures are involved as genital ridge, Wolffian (mesonephric) ducts, and Müllerian (paramesonephric) ducts [17]. Although, there is no universally accepted and interpreted classification scheme for uterine anomalies, one of the most commonly used classification scheme is the American Fertility Society Classification, which was established in 1988 [18]. Herein, we report a case of locally advanced cervical carcinoma with class V of Müllerian anomaly who underwent curative chemoradiotherapy. To the best of our knowledge, the current case is the second case of septate uterus who developed cervical cancer and was treated with chemoradiotherapy.

The first case of congenital septate uterus and locally advanced cervical carcinoma patient was reported by Platta et al. [19]. Platta et al. treated a stage IIB cervical adenocarcinoma using Rotte-Y tandem to conform to patient's septate uterus, since it was not possible to employ a standard uterine tandem. In the current case, the histopathologic subtype was squamous cell carcinoma, and a standard uterine tandem was used. The case reported by Platta et al. presented a low septate nearly abutting the internal cervical os; the current case had a septate located superiorly. Moreover, the left side of the uterus was very small. We tried to employ the tandem to both sides; however, the left side was very narrow and we could not use any applicator to the left side. Nevertheless, it was possible to achieve the suggested doses both in HR-CTV and OAR according to the GEC-ESTRO guidelines. In a recent study, it was shown that during the definitive radiotherapy for cervical cancer patients, the most expressive tumor shrinking occurs in the first three weeks of oncological treatment [20]. Platta et al. started BRT application following $23^{\text {rd }}$ fraction of EPRT in order to complete the treatment within the recommended duration [21]. In the current case, we evaluated our patient on fraction 20 of EPRT. However, we could not perform BRT application because of the remaining exophytic tumor. Nevertheless, the treatment duration was 53 days, which was in suggested duration limits.

Loo et al. reported a case of FIGO stage IIB squamous cell carcinoma with congenital abnormality of bicornuate bicollis uterus, which is a class IV Müllerian duct anomaly [17]. They inserted intra-uterine BRT applicator into both uterine cavities. Brachytherapy was performed using low-dose-rate cesium remote afterloading machine, using a Perspex vaginal cylinder applicator with a tungsten rectal shields. Cordoba et al. reported a case of locally advanced adenocarcinoma of the cervix on uterus didelphys [22]. Brachytherapy boost was performed using mold technique and a pulsed-dose-rate technique. Lee et al. reported a case of didelphys uterus, which is class III Müllerian anomaly, with FIGO stage IIA1 squamous cell carcinoma of the cervix [23]. They performed EPRT 
followed by bilateral cervical BRT, placing a tandem into each cervix and respective uterine cavity using symmetrical dual channel applicator.

The most challenging part of our treatment was tandem application. Initially, we could not find the appropriate uterine cavity. After careful examination of the patient's anatomy, we realized that the patient's septate was located superiorly, and the left side of the uterus was very small when compared to right side. Therefore, we could employ tandem to the right side. Differently from the other reported uterine anomaly cases, since the patient refused general anesthesia, we performed the application using vaginal misoprostol. Another challenge for the BRT application was the poor response of the tumor to EPRT and concomitant chemotherapy. Lastly, due to the lack of BRT applications guidelines in patients with uterine anomalies, it was difficult to perform application as well as the treatment plan.

To the best of our knowledge, the current case is the second case of septate uterus and the fifth case of uterine anomaly with locally advanced cervical cancer. Due to the rarity of uterine anomaly with locally cervical cancer patients, there is no BRT application guidelines. We don't know the most useful BRT application, the best dose-rate option in such patients. Nevertheless, we believe that sophisticated BRT techniques including 3-dimesional BRT can improve our treatment accuracy. In the current case, we preferred using standard tandem and CT-compatible ovoids, according to GEC-ESTRO guideline dose suggestions both in HR-CTV and OAR. Due to the lack of guidelines about BRT applications in patients with uterine anomaly and owing to the anatomical anomaly, BRT application is challenging in this patients population.

\section{Disclosure}

Authors report no conflict of interest.

\section{References}

1. Vargo JA, Beriwal S. Image-based brachytherapy for cervical cancer. World J Clin Oncol 2014; 5: 921-930.

2. Suneja G, Brown D, Chang A et al. American Brachytherapy Society: Brachytherapy treatment recommendations for locally advanced cervix cancer for low-income and middleincome countries. Brachytherapy 2017; 16: 85-94.

3. Murakami N, Kobayashi K, Kato T et al. The role of interstitial brachytherapy in the management of primary radiation therapy for uterine cervical cancer. J Contemp Brachytherapy 2016; 8: 391-398

4. Otter S, Franklin A, Ajaz M et al. Improving the efficiency of image guided brachytherapy in cervical cancer. J Contemp Brachytherapy 2016; 8: 557-565.

5. Small W Jr, Bacon MA, Bajaj A et al. Cervical cancer: A global health crisis. Cancer 2017; 123: 2404-2412.

6. Rose PG, Bundy BN, Watkins EB et al. Concurrent cisplatinbased radiotherapy and chemotherapy for locally advanced cervical cancer. N Engl J Med 1999; 340: 1144-1153.

7. Perez CA, Breaux S, Madoc-Jones $\mathrm{H}$ et al. Radiation therapy alone in the treatment of carcinoma of uterine cervix. I. Analysis of tumor recurrence. Cancer 1983; 51: 1393-1402.

8. Viswanathan AN, Thomadsen B; American Brachytherapy Society Cervical Cancer Recommendations Committee American Brachytherapy Society. American Brachytherapy
Society consensus guidelines for locally advanced carcinoma of the cervix. Part I: general principles. Brachytherapy 2012; 11: 33-46.

9. Potish RA. The effect of applicator geometry on dose specification in cervical cancer. Int J Radiat Oncol Biol Phys 1990; 18: 1513-1520.

10. Corn BW, Hanlon AL, Pajak TF et al. Technically accurate intracavitary insertions improve pelvic control and survival among patients with locally advanced carcinoma of the uterine cervix. Gynecol Oncol 1994; 53: 294-300.

11. Viswanathan AN, Cormack R, Rawal B et al. Increasing brachytherapy dose predicts survival for interstitial and tandem-based radiation for stage IIIB cervical cancer. Int J Gynecol Cancer 2009; 19: 1402-1406.

12. Viswanathan AN, Beriwal S, de Los Santos JF et al. American Brachytherapy Society consensus guidelines for locally advanced carcinoma of the cervix. Part II: high-dose-rate brachytherapy. Brachytherapy 2012; 11: 47-52.

13. Haie-Meder C, Pötter R, Van Limbergen E et al. Recommendations from Gynaecological (GYN) GEC-ESTRO Working Group (I): concepts and terms in 3D image based 3D treatment planning in cervix cancer brachytherapy with emphasis on MRI assessment of GTV and CTV. Radiother Oncol 2005; 74: 235-245.

14. Jurkovic D, Gruboeck K, Tailor A et al. Ultrasound screening for congenital uterine anomalies. Br J Obstet Gynaecol 1997; 104: 1320-1321.

15. Grimbizis GF, Camus M, Tarlatzis BC et al. Clinical implications of uterine malformations and hysteroscopic treatment results. Hum Reprod Update 2001; 7: 161-174.

16. Dumane VA, Yuan Y, Sheu RD et al. Computed tomography-based treatment planning for high-dose-rate brachytherapy using the tandem and ring applicator: influence of applicator choice on organ dose and inter-fraction adaptive planning. J Contemp Brachytherapy 2017; 9: 279-286.

17. Loo HW, Locks SM. Squamous cell carcinoma of the cervix report of an unusual case of bicornuate bicollis uterus treated with bilateral intracavity brachytherapy. Br J Radiol 2010; 83: e143-146.

18. The American Fertility Society classifications of adnexal adhesions, distal tubal occlusion, tubal occlusion secondary to tubal ligation, tubal pregnancies, mullerian anomalies and intrauterine adhesions. Fertil Steril 1988; 49: 944-955.

19. Platta CS, Wallace C, Gondi V et al. Cervical brachytherapy technique for locally advanced carcinoma of the cervix in a patient with septate uterus. J Contemp Brachytherapy 2014; 6: 76-81.

20. Carvalho Hde A, Mendez LC, Stuart SR et al. Implementation of image-guided brachytherapy (IGBT) for patients with uterine cervix cancer: a tumor volume kinetics approach. J Contemp Brachytherapy 2016; 8: 301-307.

21. Perez CA, Grigsby PW, Castro-Vita $\mathrm{H}$ et al. Carcinoma of the uterine cervix. I. Impact of prolongation of overall treatment time and timing of brachytherapy on outcome of radiation therapy. Int J Radiat Oncol Biol Phys 1995; 32: 1275-1288.

22. Cordoba A, Escande A, Comte P et al. Locally advanced adenocarcinoma of the cervix on uterus didelphys: a case report. J Contemp Brachytherapy 2017; 9: 71-76.

23. Lee $\mathrm{CD}$, Churn $\mathrm{M}$, Haddad $\mathrm{N}$ et al. Bilateral radical radiotherapy in a patient with uterus didelphys. Br J Radiol 2000; 73: 553-555. 